
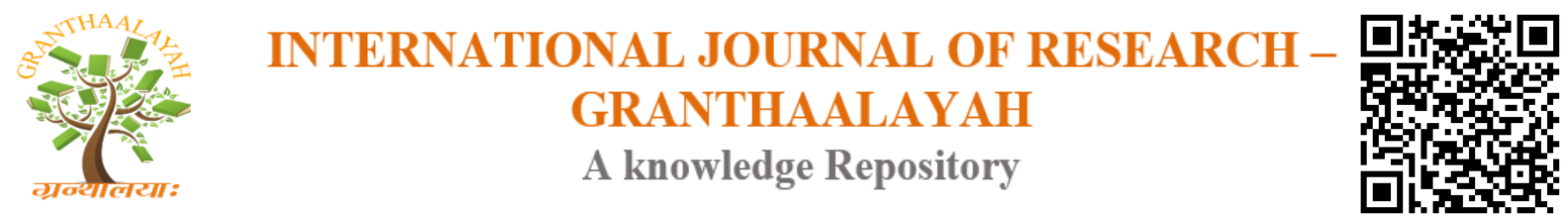

Science

\title{
ON SHOOTING AND FINITE DIFFERENCE METHODS FOR NON- LINEAR TWO POINT BOUNDARY VALUE PROBLEMS
}

\author{
Ibrahim I. O. ${ }^{1}$, Markus S. ${ }^{2}$ \\ ${ }^{1,2}$ Department of Mathematical Sciences, University of Maiduguri, Borno State, Nigeria
}

\begin{abstract}
The paper investigates the efficacy of non-linear two point boundary value problems via shooting and finite difference methods. It was observed that the shooting method provides better result as when compared to the finite difference methods with dirichlet boundary conditions. It was observed that the accuracy of the shooting method is dependent upon the integrator adopted.
\end{abstract}

Keywords: BVP; MATLAB; MAPLE; Finite Difference Method; Shooting Method; RK45; RK4; Secant Method; Newton's Method.

Cite This Article: Ibrahim I. O., and Markus S.. (2018). "ON SHOOTING AND FINITE DIFFERENCE METHODS FOR NON-LINEAR TWO POINT BOUNDARY VALUE PROBLEMS." International Journal of Research - Granthaalayah, 6(1), 23-35. https://doi.org/10.29121/granthaalayah.v6.i1.2018.1591.

\section{Introduction}

In the West, only the method defined by Euler [9] is called the Euler method, more precisely, Euler's forward method. The method defined by Henrici [8] is usually called the midpoint method and is known as the Runge method [7] or modified Euler method, which is considered as the oldest method of Runge-Kutta method characterized by the property that each step involves a multiplicity of evaluations of the right-hand side function which is sometimes called Heun's second-order method if it is predicted by Euler's forward method, and it is called the trapezoidal rule. An iterative solution of the trapezoidal rule is also considered (see [6]).

Although the Euler method [9] is not recommended in actual computation, it serves as a model for theoretical considerations and facilitates comparison with more complicated methods after Euler publishes his method.

Arieh [5] proved Cauchy convergence with the use of implicit Euler's method.

it is always better to obtain an exact solution for the given differential equations but, due to some complications like time consumption and more manual operations, it is not possible to find analytical solution for such mathematical problems. Therefore, it is necessary to approximate 
(numerical) solutions. There are so many numerical methods solving available for such differential equation such as power series method, pointwise method, Taylor's method, Picard's method, Euler's method, Improved Euler's method, Modified Euler's method, Runge-kutta second and fourth order method, predictor corrector method etc. Runge-kutta techniques have become very popular and efficient tool for computational purposes [1-4] due to many real time application problems are solved effectively. Popular RK algorithms are adopted to solve differential equations efficiently that are equivalent to approximate the exact solutions by matching ' $\mathrm{n}$ ' terms of the Taylor series expansion. Harrer [10] introduced Explicit Euler, Predictor-corrector and fourth-order Runge-kutta algorithms which are used for simulating cellulose neural works.

\section{Materials and Methods}

\subsection{Shooting Method for Non-Linear Problems}

\section{Consider}

$x^{\prime \prime}(a)=f\left(t, x(t), x^{\prime}(t)\right)$

$a \leq t \leq b$

$x(a)=A$

Where $f\left(t, x(t), x^{\prime}(t)\right)$ Is not linear in $x$ and $x^{\prime}$. Assume a given boundary value problem has a unique solution $x(t)$. we will approximate the solution $x_{(t)}$ by solving a sequence of IVPs

$x^{\prime \prime}(a)=f\left(t, x(t), x^{\prime}(t)\right)$

$a \leq t \leq b$

$x(a)=A \quad x^{\prime}(a)=s_{k}$

Where $s_{k}$ is a real number. Let $x\left(t, s_{k}\right)$ be solution of the IVP (2.2), we want to have a sequence $\left\{s_{k}\right\}$ so that

One of the choices for $S_{0}$ is

$$
\lim _{k \rightarrow \infty} x\left(t, s_{k}\right)=x(t)
$$

$s_{0}=x^{\prime}(a) \approx \frac{x(b)-x(a)}{b-a}=\frac{B-A}{b-a}$

How to choose $s_{k}$ for $k \geq 1$ ?

Consider $s$ such that

$x(b, s)-B=0$

That is $s$ is a solution of the equation. Observe that the equation $x(b, s)-B=0$ is a non-linear equation in one-variable. We can then use either Secant method or Newton method for nonlinear equation of the form

$s_{k}=s_{k-1}-\frac{g\left(s_{k-1}\right)\left(s_{k-1}-s_{k}\right)}{g\left(s_{k-1}\right)-g\left(s_{k-2}\right)}$

For $s_{0}$, the Newton's method computes 
$s_{k}=s_{k-1}-\frac{g\left(s_{k-1}\right)}{g^{\prime}\left(s_{k-1}\right)}$

These methods can be used here to solve the non-linear (2.3)

Use the secant method to approximate the solution of $x(b, s)-B=0$

$s_{k}=s_{k-1}-\frac{\left[x\left(b, s_{k-1}\right)-B\right]\left[s_{k-1}-s_{k-2}\right]}{x\left(b, s_{k-1}\right)-x\left(b, s_{k-2}\right)}$

Note that $x\left(b, S_{k-1}\right)$ is the last element in the array $x$. Note that also that we will need two initial choices $s_{0}$ and $s_{1}$ to compute $s_{2}$ in other to continue the iterations.

Use Newton's method to approximate the solution of $x(b, s)-B=0$

$s_{k}=s_{k-1}-\frac{x\left(b, s_{k-1}\right)-B}{\frac{d x}{d s}\left(b, s_{k-1}\right)}$

Again $x\left(b, S_{k-1}\right)$ is the last element in the array $x$, we do not know $x(t)$ explicitly, how we can determine $\frac{d x}{d t}\left(b, S_{k-1}\right)$ ?

Let $x(t, s)$ be the solution of the IVP (2.2). Then from (2.2), we have

$x^{\prime \prime}(t, s)=f\left(t, x_{(t, s)}, x^{\prime}(t, s) \quad a \leq t \leq b\right.$

$x(a, s)=A \quad x^{\prime}(a, s)=s$

By differentiating both sides of the equation w.r.t. s, we have

$$
\begin{aligned}
& \frac{\partial x^{\prime \prime}(t, s)}{\partial s}=\frac{\partial f\left(t, x(t, s), x^{\prime}(t, s)\right)}{\partial s} \\
& =f_{t} t_{s}+f_{x} \frac{\partial x(t, s)}{\partial s}+f_{x} \frac{\partial x^{\prime}(t, s)}{\partial s}
\end{aligned}
$$

Since $t$ and $s$ are independent, $t_{s}=0$, hence,

$\frac{\partial x \prime \prime(t, s)}{\partial s}=f_{x} \frac{\partial x(t, s)}{\partial s}+f_{x \prime} \frac{\partial x^{\prime}(t, s)}{\partial s}$

For $a \leq t \leq b$, the initial conditions are

$\frac{\partial x(a, s)}{\partial s}=\frac{d(A)}{d s}=0$

$\frac{\partial x^{\prime}(a, s)}{\partial s}=\frac{d(s)}{d s}=1$

defines $z(t, s)=\frac{d x(t, s)}{d s}$, since 


$$
\frac{\partial^{3} x(t, s)}{\partial t^{2} \partial s}=\frac{\partial}{\partial s}\left(\frac{\partial^{2} x(t, s)}{\partial t^{2}}\right)=\frac{\partial}{\partial s}\left(x^{\prime \prime}(t, s)\right)
$$

We denote

$z^{\prime \prime}(t, s)=\frac{\partial}{\partial s}\left(x^{\prime \prime}(t, s)\right)$

Then the IVP (2.4) becomes

$z^{\prime \prime}(t, s)=f_{x} z(t, s)+f_{x^{\prime}} z^{\prime}(t, s) \quad a \leq t \leq b$

$$
z(a, s)=0 \quad z^{\prime}(a, s)=1
$$

We can update our $S_{k}$ using the information from $\mathrm{z}(\mathrm{t}, \mathrm{s})$ as follows

$S_{k}=S_{k-1}-\frac{x\left(b, S_{k-1}\right)-B}{z\left(b, S_{k-1}\right)}$

\subsection{Finite Difference Method on Non-Linear Problems}

The difference method for the general non-linear boundary value problem

$x^{\prime \prime}=f\left(t, x, x^{\prime}\right)$.

for $a \leq t \leq b$, where

$x(a)=A$ and $\quad x(b)=B$

Is similar to the method applied to linear problems in last section. However, the system of equations will not be linear, so an iterative process is required to solve it.

As in the linear case, we divide $[a, b]$ into $(N+1)$ equal subintervals whose endpoints are at $x_{i}=a+i h$ for $i=0,1, \ldots, N+1$.

Assuming that the exact solution has a bounded fourth derivative allows us to replace $x^{\prime \prime}\left(t_{i}\right)$ and $x^{\prime}\left(t_{i}\right)$ in each of the equations by the appropriate centered-difference formula to obtain. For each $i=1,2, \ldots, N$,

$$
\frac{x\left(t_{i+1}\right)-2 x\left(t_{i}\right)+x\left(t_{i-1}\right)}{h^{2}}=f\left(t_{i}, x\left(t_{i}\right), \frac{x\left(t_{i+1}\right)-x\left(t_{i-1}\right)}{2 h}-\frac{h^{2}}{6} x^{\prime \prime \prime}\left(\eta_{i}\right)\right)+\frac{h^{2}}{12} x^{(4)}\left(\xi_{i}\right)
$$

For some $\left(\xi_{i}\right)$ and $\left(\eta_{i}\right)$ in the interval $\left(t_{i-1}, t_{i+1}\right)$.

The difference method results when the error terms are deleted and the boundary conditions are added. This produces the $N \times N$ non-linear system.

$$
\begin{gathered}
2 w_{1}-w_{2}+h^{2} f\left(t_{1}, w_{1}, \frac{w_{2}-A}{2 h}\right)-A=0 \\
-w_{1}+2 w_{2}-w_{3}+h^{2} f\left(t_{2}, w_{2}, \frac{w_{3}-w_{1}}{2 h}\right)=0
\end{gathered}
$$




$$
\begin{gathered}
-w_{N-2}+2 w_{N-1}-w_{N}+h^{2} f\left(t_{N-1}, w_{N-1} \frac{w_{N}-w_{N-2}}{2 h}\right)=0 \\
-w_{N-1}+2 w_{N}+h^{2} f\left(t_{N}, w_{N}, \frac{B-w_{N-1}}{2 h}\right)-B=0
\end{gathered}
$$

To approximate the solution to this system, we use Newton's method for non-linear systems, as discussed in section 2.1. A sequence of iterates.

$\left\{\left(w_{1}^{(k)}, w_{2}^{(k)}, \ldots, w_{N}^{(k)}\right)^{t}\right\}$ is generated that converges to the solution of system, provided that the initial approximation $\left(w_{1}^{(0)}, w_{2}^{(0)}, \ldots, w_{N}^{(0)}\right)^{t}$ is amply close to the true solution, $\left.\left(w_{1}, w_{2}, \ldots, w_{N}\right)^{t}\right)$.

Newton's method for nonlinear systems requires solving, at each iteration, an $N \times N$ linear system involving the Jacobian matrix. In our case, the Jacobian matrix is tri-diagonal, and Crout factorization can be applied. The initial approximations to $w_{i}^{(0)}$ to $w_{i}$ for each

$i=1,2, \ldots, N$, are obtained by passing a straight line through $(a, A)$ and $(b, B)$ and evaluating at $t_{i}$.

Since a good initial approximation may be required, an upper bound for $k$ should be specified and, if exceeded, a new initial approximation or a reduction in step size is considered.

The program was be used to solve the Nonlinear Finite Difference method on Maple as used in section 3.2.

\section{Results and Analysis}

Non - linear problem

$$
\begin{array}{rl}
x^{\prime \prime}=\frac{1}{8}\left(32+2 t^{3}-x x^{\prime}\right) & \leq t \leq 3 \\
x(1)=17 & x(3)=\frac{43}{3}
\end{array}
$$

Has an exact solution

$$
x(t)=t^{2}+\frac{16}{t}
$$

The program can be used to solve the Nonlinear Finite Difference method on Maple as shown below. $h=0.1$, to the nonlinear boundary-value problem.

\subsection{Shooting Method Solution to Non-Linear Using Rk4 as Integrator}

Suppose we want to obtain a better solution for (3.1), we shall consider more digits (i.e of $\left.\left|x\left(t_{i}\right)-w_{1, i}\right| \leq 10^{-5}\right)$, using Runge Kutta 4 , we obtain fourth initial starting value of $\mathrm{S}(4)=$ $\mathrm{S}_{3}=-14.3000203$ and when used in our fourth iteration (as in appendix) gives the following table. 
Table 1: Shooting method solution on on-linear using RK4 as integrator

\begin{tabular}{|c|c|c|c|c|c|c|c|}
\hline \multirow[t]{2}{*}{$t_{i}$} & \multirow{2}{*}{$\begin{array}{l}\begin{array}{l}\text { Analytic } \\
\text { solution }\end{array} \\
x\left(t_{i}\right)\end{array}$} & \multicolumn{2}{|c|}{$\begin{array}{l}\text { Shooting method solution } \\
\text { on non-linear problem }\end{array}$} & \multirow[t]{2}{*}{$\mathbf{t}_{\mathbf{i}}$} & \multicolumn{3}{|c|}{$\begin{array}{l}\text { Shooting method solution on nor } \\
\text { linear problem }\end{array}$} \\
\hline & & $w_{1, i}$ & $\left|x\left(t_{i}\right)-w_{1, i}\right|$ & & $x\left(t_{i}\right)$ & $w_{1, i}$ & $\left|x\left(t_{i}\right)-w_{1, i}\right|$ \\
\hline 1.0 & 17.000000 & 17.000000 & & 2.0 & 12.000000 & 12.000023 & $2.30 \times 10^{-5}$ \\
\hline 1.1 & 15.755455 & 15.755495 & $4.06 \times 10^{-5}$ & 2.1 & 12.029048 & 12.029066 & $2.88 \times 10^{-5}$ \\
\hline 1.2 & 14.773333 & 14.773389 & $5.60 \times 10^{-5}$ & 2.2 & 12.112727 & 12.112741 & $4.17 \times 10^{-5}$ \\
\hline 1.3 & 13.997692 & 13.997752 & $5.94 \times 10^{-5}$ & 2.3 & 12.246522 & 12.246532 & $4.55 \times 10^{-5}$ \\
\hline 1.4 & 13.388571 & 13.388629 & $5.71 \times 10^{-5}$ & 2.4 & 12.426667 & 12.426673 & $4.39 \times 10^{-5}$ \\
\hline 1.5 & 12.916667 & 12.916719 & $5.23 \times 10^{-5}$ & 2.5 & 12.650000 & 12.650004 & $3.92 \times 10^{-5}$ \\
\hline 1.6 & 12.560000 & 12.560046 & $4.64 \times 10^{-5}$ & 2.6 & 12.913845 & 12.913847 & $3.26 \times 10^{-5}$ \\
\hline 1.7 & 12.301765 & 12.301805 & $4.02 \times 10^{-5}$ & 2.7 & 13.215926 & 13.215924 & $2.49 \times 10^{-5}$ \\
\hline 1.8 & 12.128889 & 12.128923 & $3.14 \times 10^{-5}$ & 2.8 & 13.554286 & 13.554284 & $1.68 \times 10^{-5}$ \\
\hline 1.9 & 12.031053 & 12.031081 & $2.84 \times 10^{-5}$ & 2.9 & 13.927241 & 13.927236 & $8.41 \times 10^{-5}$ \\
\hline & & & & 3.0 & 14.333333 & 14.333327 & \\
\hline
\end{tabular}

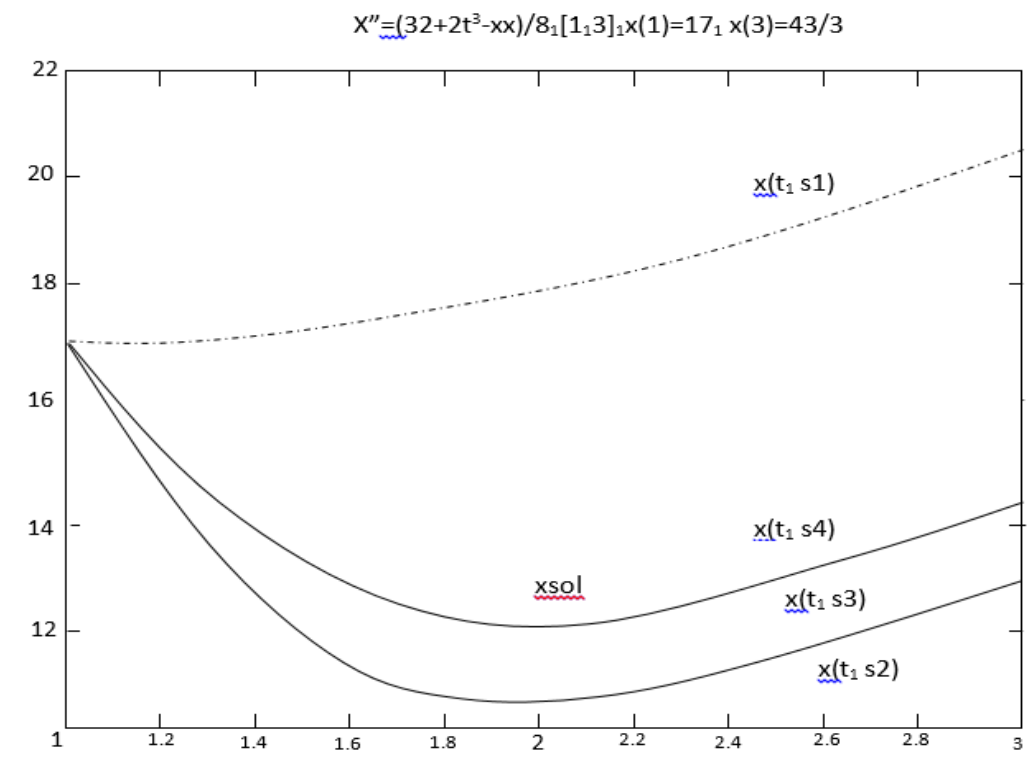

Graph 2: showing Figure shooting method solution to non-linear

Comparing our result of table we observe that RK4 gives a better approximation to the problem than the embedded RK4 since RK4 produces a smaller error (of $\left|x\left(t_{i}\right)-w_{1, i}\right| \leq 10^{-5}$ ) when compared to the actual solution.

\subsection{Finite Difference Method Solution to Non - Linear Problems}

Suppose we want to obtain a solution of $\left|x\left(t_{i}\right)-w_{1, i}\right| \leq 10^{-3}$, for (3.1) via the non - linear 
Table 2: Finite Difference Method Solution On Non-Linear

\begin{tabular}{|c|c|c|c|c|c|c|c|}
\hline \multirow{2}{*}{$t_{\bar{i}}$} & \multirow{2}{*}{$\begin{array}{l}\begin{array}{l}\text { Analytic } \\
\text { solution }\end{array} \\
x\left(t_{i}\right)\end{array}$} & \multicolumn{2}{|c|}{ finite difference method solution } & \multirow{2}{*}{$t_{\bar{k}}$} & \multicolumn{3}{|c|}{ finite difference method solution } \\
\hline & & $w_{t, i}$ & $\left|x\left(t_{i}\right)-w_{1, i}\right|$ & & $x\left(t_{i}\right)$ & $w_{1, i}$ & ||$x\left(t_{i j}\right)-w_{1, i} \mid$ \\
\hline 1.0 & 17.000000 & 17.000000 & & 2.0 & 12.000000 & 11.997915 & $2.085 \times 10^{-3}$ \\
\hline 1.1 & 15.755455 & 15.754503 & $9.520 \times 10^{-4}$ & 2.1 & 12.029048 & 12.027142 & $1.905 \times 10^{-3}$ \\
\hline 1.2 & 14.773333 & 14.771740 & $1.594 \times 10^{-3}$ & 2.2 & 12.112727 & 12.111020 & $1.707 \times 10^{-3}$ \\
\hline 1.3 & 13.997692 & 13.995677 & $2.015 \times 10^{-3}$ & 2.3 & 12.246522 & 12.245025 & $1.497 \times 10^{-3}$ \\
\hline 1.4 & 13.388571 & 13.386297 & $2.275 \times 10^{-3}$ & 2.4 & 12.426667 & 12.425388 & $1.278 \times 10^{-3}$ \\
\hline 1.5 & 12.916667 & 12.914252 & $2.414 \times 10^{-3}$ & 2.5 & 12.650000 & 12.648944 & $1.056 \times 10^{-3}$ \\
\hline 1.6 & 12.560000 & 12.557538 & $2.462 \times 10^{-3}$ & 2.6 & 12.913845 & 12.913013 & $8.335 \times 10^{-4}$ \\
\hline 1.7 & 12.301765 & 12.299326 & $2.438 \times 10^{-3}$ & 2.7 & 13.215926 & 13.215312 & $6.142 \times 10^{-4}$ \\
\hline 1.8 & 12.128889 & 12.126529 & $2.360 \times 10^{-3}$ & 2.8 & 13.554286 & 13.553885 & $4.006 \times 10^{-4}$ \\
\hline \multirow[t]{2}{*}{1.9} & 12.031053 & 12.028814 & $2.239 \times 10^{-3}$ & 2.9 & 13.927241 & 13.927046 & $1.953 \times 10^{-4}$ \\
\hline & & & & 3.0 & 14.333333 & 14.333333 & \\
\hline
\end{tabular}

Finite difference method with four iterations produces the result in the table below.

Table 3: Comparing finite difference method and shooting method solution to Non-Linear

\begin{tabular}{|l|l|l|l|l|l|}
\hline \multirow{2}{*}{$\boldsymbol{t}_{\boldsymbol{i}}$} & Analytic solution & \multicolumn{2}{|l|}{ finite difference method solution } & \multicolumn{2}{|c|}{ shooting method solution } \\
\cline { 2 - 6 } & $\boldsymbol{x}\left(\boldsymbol{t}_{\boldsymbol{i}}\right)$ & $\boldsymbol{w}_{\boldsymbol{1}, \boldsymbol{i}}$ & $\left|\boldsymbol{x}\left(\boldsymbol{t}_{\boldsymbol{i}}\right)-\boldsymbol{w}_{\boldsymbol{1}, \boldsymbol{i}}\right|$ & $\boldsymbol{w}_{\boldsymbol{i}}$ & $\left|\boldsymbol{x}\left(\boldsymbol{t}_{\boldsymbol{i}}\right)-\boldsymbol{w}_{\boldsymbol{i}}\right|$ \\
\hline 1.0 & 17.000000 & 17.000000 & & 17.000000 & \\
\hline .1 .1 & 15.755455 & 15.754503 & $9.520 \times 10^{-4}$ & 15.755495 & $4.06 \times 10^{-5}$ \\
\hline 1.2 & 14.773333 & 14.771740 & $1.594 \times 10^{-3}$ & 14.773389 & $5.60 \times 10^{-5}$ \\
\hline 1.3 & 13.997692 & 13.995677 & $2.015 \times 10^{-3}$ & 13.997752 & $5.94 \times 10^{-5}$ \\
\hline 1.4 & 13.388571 & 13.386297 & $2.275 \times 10^{-3}$ & 13.388629 & $5.71 \times 10^{-5}$ \\
\hline 1.5 & 12.916667 & 12.914252 & $2.414 \times 10^{-3}$ & 12.916719 & $5.23 \times 10^{-5}$ \\
\hline 1.6 & 12.560000 & 12.557538 & $2.462 \times 10^{-4}$ & 12.560046 & $4.64 \times 10^{-5}$ \\
\hline 1.7 & 12.301765 & 12.299326 & $2.438 \times 10^{-3}$ & 12.301805 & $4.02 \times 10^{-5}$ \\
\hline 1.8 & 12.128889 & 12.126529 & $2.360 \times 10^{-3}$ & 12.128923 & $3.14 \times 10^{-5}$ \\
\hline 1.9 & 12.031053 & 12.028814 & $2.239 \times 10^{-3}$ & 12.031081 & $2.84 \times 10^{-5}$ \\
\hline 2.0 & 12.000000 & 11.997915 & $2.085 \times 10^{-3}$ & 12.000023 & $2.30 \times 10^{-5}$ \\
\hline 2.1 & 12.029048 & 12.027142 & $1.905 \times 10^{-3}$ & 12.029066 & $2.88 \times 10^{-5}$ \\
\hline 2.2 & 12.112727 & 12.111020 & $1.707 \times 10^{-3}$ & 12.112741 & $4.17 \times 10^{-5}$ \\
\hline 2.3 & 12.246522 & 12.245025 & $1.497 \times 10^{-3}$ & 12.246532 & $4.55 \times 10^{-5}$ \\
\hline 2.4 & 12.426667 & 12.425388 & $1.278 \times 10^{-3}$ & 12.426673 & $4.39 \times 10^{-5}$ \\
\hline 2.5 & 12.650000 & 12.648944 & $1.056 \times 10^{-3}$ & 12.650004 & $3.92 \times 10^{-5}$ \\
\hline 2.6 & 12.913845 & 12.913013 & $8.335 \times 10^{-4}$ & 12.913847 & $3.26 \times 10^{-5}$ \\
\hline
\end{tabular}




\begin{tabular}{|l|l|l|l|l|l|}
\hline 2.7 & 13.215926 & 13.215312 & $6.142 \times 10^{-4}$ & 13.215924 & $2.49 \times 10^{-5}$ \\
\hline 2.8 & 13.554286 & 13.553885 & $4.006 \times 10^{-4}$ & 13.554284 & $1.68 \times 10^{-5}$ \\
\hline 2.9 & 13.927241 & 13.927046 & $1.953 \times 10^{-4}$ & 13.927236 & $8.41 \times 10^{-5}$ \\
\hline 3.0 & 14.333333 & 14.333333 & & 14.333327 & \\
\hline
\end{tabular}

\section{Conclusion}

The results from the finite difference method are considerable less accurate than those obtained using the shooting method. This is because the shooting method used involves a Runge-Kutta technique with error of order $O\left(h^{4}\right)$ whereas the difference method used here has error of order $O\left(h^{2}\right)$.

To obtain a difference method with greater accuracy, we can proceed in a number of ways. Using fifth-order Taylor series for approximating $x^{\prime \prime}\left(t_{i}\right)$ and $x^{\prime}\left(t_{i}\right)$ result in an error term involving $\mathrm{h}^{4}$. i.e.

Central difference approximation for $1^{\text {st }}$ derivative, $4^{\text {th }}$ order

$\left|\frac{d x}{d t}\right|_{i}=\frac{-x_{i+2}+8 x_{i+1}-8 x_{i-1}+x_{i-2}}{12 h}+O\left(h^{4}\right)$

Central difference approximation for $2^{\text {nd }}$ derivative, $4^{\text {th }}$ order

$\left|\frac{d^{2} x}{d t^{2}}\right|_{i}=\frac{-x_{i+2}+16 x_{i+1}-30 x_{i}+16 x_{i-2}-x_{i-2}}{12 h^{2}}+O\left(h^{4}\right)$

Using this approximation leads to difficulty at $i=0$ and $i=N$. Moreover, the resulting solution of equations is not in tri-diagonal form and the solution to the system requires many more calculations. Instead of obtaining a difference method with a higher-order error term in this manner, it is generally more satisfactory to consider a reduction in the step size.

\section{APPENDIX A}

Table 4: First Shot Using the First Guessed Value of -1.333 in Providing Solution to Non-Linear Using Integrator RK45

\begin{tabular}{|lcccc|lllll|}
\hline $\mathrm{t}$ & $\mathrm{x}$ & $\mathrm{X}^{1}$ & $\mathrm{Z}$ & $\mathrm{Z}^{\prime}$ & $\mathrm{t}$ & $\mathrm{x}$ & $\mathrm{X}^{\prime}$ & $\mathrm{Z}$ & $\mathrm{Z}^{\prime}$ \\
& $\mathbf{U}_{\mathbf{1}}(\mathbf{1})$ & $\mathbf{U}_{\mathbf{1}}(\mathbf{1})$ & $\mathbf{U}_{\mathbf{3}}(\mathbf{1})$ & $\mathbf{U}_{\mathbf{4}}(\mathbf{1})$ & & $\mathbf{U}_{\mathbf{1}}(\mathbf{1})$ & $\mathbf{U}_{\mathbf{1}}(\mathbf{1})$ & $\mathbf{U}_{\mathbf{3}}(\mathbf{1})$ & $\mathbf{U}_{\mathbf{4}} \mathbf{( 1 )}$ \\
1.0000 & 17.0000 & -1.3333 & 0 & 1.0000 & 1.7069 & 17.2393 & 1.4504 & 0.3655 & 0.2125 \\
1.0001 & 16.9999 & -1.3330 & 0.0001 & 0.9999 & 1.7569 & 17.3144 & 1.5531 & 0.3754 & 0.1874 \\
1.0001 & 16.9999 & -1.3326 & 0.0001 & 0.9998 & 1.8069 & 17.3945 & 1.6501 & 0.3842 & 0.1645 \\
1.0002 & 16.9998 & -1.3323 & 0.0002 & 0.9997 & 1.8569 & 17.4794 & 1.7421 & 0.3919 & 0.1437 \\
1.0002 & 16.9997 & -1.3319 & 0.0002 & 0.9996 & 1.9069 & 17.5687 & 1.8298 & 0.3986 & 0.1246 \\
1.0005 & 16.9994 & -1.3301 & 0.0005 & 0.9990 & 1.9569 & 17.6623 & 1.9138 & 0.4044 & 0.1071 \\
1.0007 & 16.9991 & -1.3284 & 0.0007 & 0.9985 & 2.0069 & 17.7600 & 1.9948 & 0.4094 & 0.0912 \\
1.0010 & 16.9987 & -1.3266 & 0.0010 & 0.9980 & 2.0569 & 17.8617 & 2.0732 & 0.4136 & 0.0766 \\
1.0012 & 16.9984 & -1.3248 & 0.0012 & 0.9974 & 2.1069 & 17.9673 & 2.1496 & 0.4171 & 0.0633 \\
1.0025 & 16.9967 & -1.3159 & 0.0025 & 0.9948 & 2.1569 & 18.0767 & 2.2244 & 0.4199 & 0.0512 \\
\hline
\end{tabular}


[Ibrahim et. al., Vol.6 (Iss.1): January, 2018]

(Received: Dec 28, 2017 - Accepted: Jan 18, 2018)
ISSN- 2350-0530(O), ISSN- 2394-3629(P) DOI: 10.5281/zenodo.1162064

\begin{tabular}{|lllll|lllll|}
\hline 1.0037 & 16.9951 & -1.3071 & 0.0037 & 0.9921 & 2.2069 & 18.1897 & 2.2980 & 0.4222 & 0.0401 \\
1.0050 & 16.9935 & -1.2983 & 0.0049 & 0.9895 & 2.2569 & 18.3064 & 2.3708 & 0.4239 & 0.0299 \\
1.0062 & 16.9918 & -1.2895 & 0.0062 & 0.9869 & 2.3069 & 18.4268 & 2.4430 & 0.4252 & 0.0206 \\
1.0125 & 16.9839 & -1.2459 & 0.0123 & 0.9738 & 2.3569 & 18.5507 & 2.5151 & 0.4260 & 0.0121 \\
1.0188 & 16.9762 & -1.2028 & 0.0184 & 0.9609 & 2.4069 & 18.6783 & 2.5872 & 0.4264 & 0.0044 \\
1.0251 & 16.9688 & -1.1602 & 0.0244 & 0.9482 & 2.4569 & 18.8095 & 2.6597 & 0.4265 & -0.0027 \\
1.0313 & 16.9616 & -1.1182 & 0.0303 & 0.9357 & 2.5069 & 18.9443 & 2.7327 & 0.4262 & -0.0092 \\
1.0627 & 16.9297 & -0.9161 & 0.0588 & 0.8757 & 2.5569 & 19.0827 & 2.8065 & 0.4256 & -0.0151 \\
1.0941 & 16.9040 & -0.7262 & 0.0854 & 0.8196 & 2.6069 & 19.2249 & 2.8813 & 0.4247 & -0.0205 \\
1.1255 & 16.8840 & -0.5477 & 0.1103 & 0.7673 & 2.6569 & 19.3709 & 2.9571 & 0.4235 & -0.0255 \\
1.1569 & 16.8695 & -0.3797 & 0.1336 & 0.7183 & 2.7069 & 19.5207 & 3.0342 & 0.4221 & -0.0300 \\
1.2069 & 16.8567 & -0.1323 & 0.1677 & 0.6467 & 2.7569 & 19.6743 & 3.1127 & 0.4205 & -0.0342 \\
1.2569 & 16.8558 & 0.0930 & 0.1984 & 0.5820 & 2.8069 & 19.8320 & 3.1926 & 0.4187 & -0.0380 \\
1.3069 & 16.8657 & 0.2985 & 0.2260 & 0.5236 & 2.8569 & 19.9936 & 3.2741 & 0.4167 & -0.0415 \\
1.3569 & 16.8854 & 0.4865 & 0.2508 & 0.4706 & 2.9069 & 20.1594 & 3.3573 & 0.4146 & -0.0447 \\
1.4069 & 16.9141 & 0.6589 & 0.2731 & 0.4225 & 2.9569 & 20.3294 & 3.4423 & 0.4123 & -0.0476 \\
1.4569 & 16.9511 & 0.8174 & 0.2931 & 0.3789 & 2.9677 & 20.3665 & 3.4608 & 0.4117 & -0.0482 \\
1.5069 & 16.9956 & 0.9635 & 0.3111 & 0.3391 & 2.9785 & 20.4039 & 3.4794 & 0.4112 & -0.0488 \\
1.5569 & 17.0472 & 1.0987 & 0.3271 & 0.3030 & 2.9892 & 20.4414 & 3.4980 & 0.4107 & -0.0494 \\
1.6069 & 17.1053 & 1.2241 & 0.3414 & 0.2700 & 3.0000 & 20.4792 & 3.5168 & 0.4102 & -0.0500 \\
1.6569 & 17.1695 & 1.3410 & 0.3542 & 0.2399 & & & & & \\
\hline
\end{tabular}

\section{APPENDIX B}

Table 5: Second shot using the second starting value of -16.3174 in providing solution to nonlinear using integrator RK45

\begin{tabular}{|lcccl|lllll|}
\hline$t$ & $x$ & $x^{\prime}$ & $z$ & $z^{\prime}$ & $t$ & $x$ & $x^{\prime}$ & $z$ & $z^{\prime}$ \\
1.0000 & 17.0000 & -16.3174 & 0 & 1.0000 & 1.7069 & 11.3030 & -2.9441 & 0.4305 & 0.3918 \\
10001 & 16.9992 & -16.3155 & 0.0001 & 0.9999 & 1.7569 & 11.1673 & -2.4886 & 0.4496 & 0.3724 \\
1.0001 & 16.9984 & -16.3135 & 0.0001 & 0.9998 & 1.8069 & 11.0537 & -2.0600 & 0.4677 & 0.3537 \\
1.0002 & 16.9975 & -16.3116 & 0.0002 & 0.9997 & 1.8569 & 10.9609 & -1.6555 & 0.4850 & 0.3356 \\
1.0002 & 16.9967 & -16.3096 & 0.0002 & 0.9996 & 1.9069 & 10.8878 & -1.2723 & 0.5013 & 0.3178 \\
1.0005 & 16.9926 & -16.2999 & 0.0005 & 0.9990 & 1.9569 & 10.8333 & -0.9083 & 0.5168 & 0.3003 \\
1.0007 & 16.9885 & -16.2901 & 0.0007 & 0.9985 & 2.0069 & 10.7966 & -0.5614 & 0.5313 & 0.2829 \\
1.0010 & 16.9844 & -16.2803 & 0.0010 & 0.9980 & 2.0569 & 10.7769 & -0.2299 & 0.5451 & 0.2658 \\
1.0012 & 16.9804 & -16.2706 & 0.0012 & 0.9974 & 2.1069 & 10.7734 & 0.0876 & 0.5579 & 0.2487 \\
1.0025 & 16.9599 & -16.2220 & 0.0025 & 0.9948 & 2.1569 & 10.7855 & 0.3925 & 0.5699 & 0.2317 \\
1.0037 & 16.9396 & -16.1735 & 0.0037 & 0.9922 & 2.2069 & 10.8125 & 0.6859 & 0.5811 & 0.2147 \\
1.0050 & 16.9193 & -16.1253 & 0.0049 & 0.9895 & 2.2569 & 10.8539 & 0.9689 & 0.5914 & 0.1977 \\
1.0062 & 16.8991 & -16.0772 & 0.0062 & 0.9869 & 2.3069 & 10.9092 & 1.2422 & 0.6008 & 0.1807 \\
1.0125 & 16.7989 & -15.8394 & 0.0123 & 0.9741 & 2.3569 & 10.9780 & 1.5067 & 0.6095 & 0.1637 \\
1.0188 & 16.7002 & -15.6059 & 0.0184 & 0.9615 & 2.4069 & 11.0597 & 1.7630 & 0.6172 & 0.1467 \\
1.0251 & 16.6029 & -15.3766 & 0.0244 & 0.9493 & 2.4569 & 11.1541 & 2.0117 & 0.6241 & 0.1298 \\
1.0313 & 16.5070 & -15.1515 & 0.0303 & 0.9374 & 2.5069 & 11.2608 & 2.2534 & 0.6302 & 0.1130 \\
1.0627 & 16.0483 & -14.0834 & 0.0589 & 0.8819 & 2.5569 & 11.3794 & 2.4886 & 0.6354 & 0.0962 \\
1.0941 & 15.6217 & -13.1036 & 0.0858 & 0.8325 & 2.6069 & 11.5096 & 2.7175 & 0.6398 & 0.0795 \\
1.1255 & 15.2246 & -12.2018 & 0.1112 & 0.7883 & 2.6569 & 11.6510 & 2.9406 & 0.6434 & 0.0630 \\
1.1569 & 14.8547 & -11.3692 & 0.1353 & 0.7487 & 2.7069 & 11.8035 & 3.1582 & 0.6461 & 0.0467 \\
1.2069 & 14.3169 & -10.1678 & 0.1714 & 0.6933 & 2.7569 & 11.9668 & 3.3706 & 0.6481 & 0.0306 \\
\hline
\end{tabular}


[Ibrahim et. al., Vol.6 (Iss.1): January, 2018]

(Received: Dec 28, 2017 - Accepted: Jan 18, 2018)

ISSN- 2350-0530(O), ISSN- 2394-3629(P) DOI: 10.5281/zenodo.1162064

\begin{tabular}{|ccccc|ccccc|}
\hline 1.2569 & 13.8357 & -9.0978 & 0.2048 & 0.6458 & 2.8069 & 12.1405 & 3.5780 & 0.6492 & 0.0148 \\
1.3069 & 13.4053 & -8.1387 & 0.2360 & 0.6045 & 2.8569 & 12.3245 & 3.7806 & 0.6495 & -0.0006 \\
1.3569 & 13.0203 & -7.2735 & 0.2653 & 0.5682 & 2.9069 & 12.5185 & 3.9786 & 0.6491 & -0.0157 \\
1.4069 & 12.6766 & -6.4884 & 0.2929 & 0.5359 & 2.9569 & 12.7223 & 4.1722 & 0.6480 & -0.0304 \\
1.4569 & 12.3703 & -5.7723 & 0.3190 & 0.5068 & 2.9677 & 12.7674 & 4.2133 & 0.6476 & -0.0336 \\
1.5069 & 12.0984 & -5.1157 & 0.3436 & 0.4803 & 2.9785 & 12.8130 & 4.2542 & 0.6473 & -0.0366 \\
1.5569 & 11.8579 & -4.5107 & 0.3670 & 0.4560 & 2.9892 & 12.8590 & 4.2949 & 0.6468 & -0.0397 \\
1.6069 & 11.6466 & -3.9507 & 0.3893 & 0.4333 & 3.0000 & 12.9054 & 4.3355 & 0.6464 & -0.0427 \\
1.6569 & 11.4622 & -3.4301 & 0.4104 & 0.4120 & & & & & \\
\hline
\end{tabular}

\section{APPENDIX C}

Table 6: Third shot using the third starting value of -14.1085 in providing solutions to non-linear using integrator RK45

\begin{tabular}{|c|c|c|c|c|c|c|c|c|c|}
\hline$t$ & $\begin{array}{c}x \\
u_{1}(3)\end{array}$ & $\begin{array}{c}x^{\prime} \\
u_{1}(3)\end{array}$ & $\begin{array}{c}z \\
u_{3}(3)\end{array}$ & $\begin{array}{c}z^{\prime} \\
u_{4}(3)\end{array}$ & $t$ & $\begin{array}{c}x \\
u_{1}(3)\end{array}$ & $\begin{array}{c}x^{\prime} \\
u_{1}(3)\end{array}$ & $\begin{array}{c}z \\
u_{3}(3)\end{array}$ & $\begin{array}{c}z^{\prime} \\
u_{4}(3)\end{array}$ \\
\hline 1.0000 & 17.0000 & -14.1085 & 0 & 1.0000 & 1.7069 & 12.2416 & -2.1164 & 0.4195 & 0.3581 \\
\hline 1.0001 & 16.9993 & -14.1067 & 0.0001 & 0.9999 & 1.7569 & 12.1462 & -1.7059 & 0.4368 & 0.3368 \\
\hline 1.0001 & 16.9986 & -14.1050 & 0.0001 & 0.9998 & 1.8069 & 12.0706 & -1.3208 & 0.4532 & 0.3163 \\
\hline 1.0002 & 16.9979 & -14.1033 & 0.0002 & 0.9997 & 1.8569 & 12.0137 & -0.9583 & 0.4685 & 0.2965 \\
\hline 1.0002 & 16.9972 & -14.1016 & 0.0002 & 0.9996 & 1.9069 & 11.9745 & -0.6161 & 0.4828 & 0.2773 \\
\hline 1.0005 & 16.9936 & -14.0930 & 0.0005 & 0.9990 & 1.9569 & 11.9518 & -0.2921 & 0.4962 & 0.2587 \\
\hline 1.0007 & 16.9901 & -14.0844 & 0.0007 & 0.9985 & 2.0069 & 11.9450 & 0.0155 & 0.5087 & 0.2405 \\
\hline 1.0010 & 16.9865 & -14.0758 & 0.0010 & 0.9980 & 2.0569 & 11.9531 & 0.3082 & 0.5203 & 0.2227 \\
\hline 1.0012 & 16.9830 & -14.0673 & 0.0012 & 0.9974 & 2.1069 & 11.9756 & 0.5874 & 0.5310 & 0.2052 \\
\hline 1.0025 & 16.9654 & -14.0245 & 0.0025 & 0.9948 & 2.1569 & 12.0117 & 0.8545 & 0.5408 & 0.1880 \\
\hline 1.0037 & 16.9478 & -13.9819 & 0.0037 & 0.9922 & 2.2069 & 12.0608 & 1.1104 & 0.5498 & 0.1712 \\
\hline 1.0050 & 16.9303 & -13.9394 & 0.0049 & 0.9895 & 2.2569 & 12.1225 & 1.3561 & 0.5579 & 0.1546 \\
\hline 1.0062 & 16.9128 & -13.8971 & 0.0062 & 0.9869 & 2.3069 & 12.1963 & 1.5925 & 0.5652 & 0.1383 \\
\hline 1.0125 & 16.8262 & -13.6877 & 0.0123 & 0.9740 & 2.3569 & 12.2817 & 1.8205 & 0.5718 & 0.1223 \\
\hline 1.0188 & 16.7409 & -13.4820 & 0.0184 & 0.9614 & 2.4069 & 12.3782 & 2.0406 & 0.5775 & 0.1065 \\
\hline 1.0251 & 16.6568 & -13.2798 & 0.0244 & 0.9492 & 2.4569 & 12.4856 & 2.2536 & 0.5824 & 0.0911 \\
\hline 1.0313 & 16.5741 & -13.0811 & 0.0303 & 0.9371 & 2.5069 & 12.6035 & 2.4599 & 0.5866 & 0.0759 \\
\hline 1.0627 & 16.1784 & -12.1364 & 0.0589 & 0.8809 & 2.5569 & 12.7315 & 2.6601 & 0.5900 & 0.0611 \\
\hline 1.0941 & 15.8111 & -11.2668 & 0.0857 & 0.8306 & 2.6069 & 12.8694 & 2.8546 & 0.5927 & 0.0466 \\
\hline 1.1255 & 15.4701 & -10.4638 & 0.1111 & 0.7852 & 2.6569 & 13.0169 & 3.0439 & 0.5947 & 0.0324 \\
\hline 1.1569 & 15.1534 & -9.7204 & 0.1351 & 0.7441 & 2.7069 & 13.1737 & 3.2284 & 0.5959 & 0.0187 \\
\hline 1.2069 & 14.6948 & -8.6440 & 0.1708 & 0.6862 & 2.7569 & 13.3396 & 3.4083 & 0.5965 & 0.0053 \\
\hline 1.2569 & 14.2870 & -7.6821 & 0.2038 & 0.6360 & 2.8069 & 13.5144 & 3.5840 & 0.5965 & 0.0076 \\
\hline 1.3069 & 13.9250 & -6.8175 & 0.2345 & 0.5918 & 2.8569 & 3.6980 & 3.7558 & 0.5958 & -0.0201 \\
\hline 1.3569 & 13.6040 & -6.0357 & 0.2631 & 0.5526 & 2.9069 & 13.8900 & 3.9240 & 0.5945 & -0.0321 \\
\hline 1.4069 & 13.3202 & -5.3251 & 0.2898 & 0.5174 & 2.9569 & 14.0903 & 4.0887 & 0.5926 & -0.0437 \\
\hline 1.4569 & 13.0704 & -4.6764 & 0.3149 & 0.4855 & 2.9677 & 14.1345 & 4.1238 & 0.5921 & -0.0461 \\
\hline 1.5069 & 12.8517 & -4.0814 & 0.3384 & 0.4563 & 2.9785 & 14.1791 & 4.1587 & 0.5916 & -0.0485 \\
\hline 1.5569 & 12.6615 & -3.5332 & 0.3606 & 0.4294 & 2.9892 & 14.2240 & 4.1934 & 0.5911 & -0.0509 \\
\hline 1.6069 & 12.4977 & -3.0261 & 0.3814 & 0.4042 & 3.0000 & 14.2693 & 4.2280 & 0.5905 & -0.0530 \\
\hline 1.6569 & 12.3583 & -2.5552 & 0.4010 & 0.3805 & & & & & \\
\hline
\end{tabular}




\section{APPENDIX D}

Table 7: Fourth shot using the Fourth starting value of -14.0001 in providing solution to nonlinear using integrator RK45

\begin{tabular}{|c|c|c|c|c|c|c|c|c|c|}
\hline$t$ & $\begin{array}{r}x \\
u_{1}(4)\end{array}$ & $\begin{array}{c}x^{\prime} \\
u_{1}(4)\end{array}$ & $\begin{array}{c}z \\
u_{3}(4)\end{array}$ & $\begin{array}{c}z^{\prime} \\
u_{4}(4)\end{array}$ & $t$ & $\begin{array}{r}x \\
u_{1}(4)\end{array}$ & $\begin{array}{c}x^{\prime} \\
u_{1}(4)\end{array}$ & $\begin{array}{l}z \\
u_{3}(4)\end{array}$ & $\begin{array}{l}z^{\prime} \\
u_{4}(4)\end{array}$ \\
\hline 1.0000 & 17.0000 & -14.0001 & 0 & 1.0000 & 1.7069 & 12.2871 & -2.0776 & 0.4189 & 0.3566 \\
\hline 1.0001 & 16.9993 & -13.9984 & 0.0001 & 0.9999 & 1.7569 & 12.1935 & -1.6695 & 0.4362 & 0.3351 \\
\hline 1.0001 & 16.9986 & -13.9967 & 0.0001 & 0.9998 & 1.8569 & 12.0645 & -0.9263 & 0.4677 & 0.2947 \\
\hline 1.0002 & 16.9979 & -13.9950 & 0.0002 & 0.9997 & 1.9069 & 12.0267 & -0.5861 & 0.4820 & 0.2755 \\
\hline 1.0002 & 16.9972 & -13.9933 & 0.0002 & 0.9996 & 1.9569 & 12.0056 & -0.2642 & 0.4953 & 0.2568 \\
\hline 1.0005 & 16.9937 & -13.9847 & 0.0005 & 0.9990 & 2.0069 & 12.0000 & 0.0414 & 0.5076 & 0.2386 \\
\hline 1.0007 & 16.9902 & -13.9762 & 0.0007 & 0.9985 & 2.0569 & 12.0094 & 0.3322 & 0.5191 & 0.2207 \\
\hline 1.0010 & 16.9867 & -13.9677 & 0.0010 & 0.9980 & 2.1069 & 12.0330 & 0.6096 & 0.5297 & 0.2033 \\
\hline 1.0012 & 16.9831 & -13.9592 & 0.0012 & 0.9974 & 2.1569 & 12.0702 & 0.8747 & 0.5395 & 0.1861 \\
\hline 1.0025 & 16.9656 & -13.9167 & 0.0025 & 0.9948 & 2.2069 & 12.1203 & 1.1288 & 0.5483 & 0.1693 \\
\hline 1.0037 & 16.9482 & -13.8744 & 0.0037 & 0.9922 & 2.2569 & 12.1829 & 1.3727 & 0.5564 & 0.1527 \\
\hline 1.0050 & 16.9308 & -13.8322 & 0.0049 & 0.9895 & 2.3069 & 12.2575 & 1.6074 & 0.5636 & 0.1365 \\
\hline 1.0062 & 16.9134 & -13.7901 & 0.0062 & 0.9869 & 2.3569 & 12.3435 & 1.8336 & 0.5700 & 0.1205 \\
\hline 1.0125 & 16.8275 & -13.5822 & 0.0123 & 0.9740 & 2.4069 & 12.4407 & 2.0521 & 0.5757 & 0.1048 \\
\hline 1.0188 & 16.7429 & -13.3778 & 0.0184 & 0.9614 & 2.4569 & 12.5486 & 2.2633 & 0.5805 & 0.0894 \\
\hline 1.0251 & 16.6595 & -13.1769 & 0.0244 & 0.9491 & 2.5069 & 12.6669 & 2.4680 & 0.5846 & 0.0744 \\
\hline 1.0313 & 16.5773 & -12.9795 & 0.0303 & 0.9371 & 2.5569 & 12.7953 & 2.6666 & 0.5880 & 0.0596 \\
\hline 1.0627 & 16.1847 & -12.0410 & 0.0589 & 0.8809 & 2.6069 & 12.9335 & 2.8596 & 0.5906 & 0.0452 \\
\hline 1.0941 & 15.8204 & -11.1768 & 0.0857 & 0.8305 & 2.6569 & 13.0812 & 3.0474 & 0.5925 & 0.0312 \\
\hline 1.1255 & 15.4822 & -10.3787 & 0.1111 & 0.7850 & 1.8069 & 12.1197 & -1.2866 & 0.4525 & 0.3145 \\
\hline 1.1569 & 15.1681 & -9.6397 & 0.1351 & 0.7439 & 2.7069 & 13.2381 & 3.2303 & 0.5937 & 0.0176 \\
\hline 1.2069 & 14.7133 & -8.5697 & 0.1708 & 0.6859 & 2.7569 & 13.4041 & 3.4088 & 0.5943 & 0.0043 \\
\hline 1.2569 & 14.3091 & -7.6133 & 0.2038 & 0.6355 & 2.8069 & 13.5789 & 3.5831 & 0.5941 & -0.0085 \\
\hline 1.3069 & 13.9504 & -6.7534 & 0.2344 & 0.5912 & 2.8569 & 13.7624 & 3.7536 & 0.5934 & -0.0208 \\
\hline 1.3569 & 13.6325 & -5.9758 & 0.2630 & 0.5519 & 2.9069 & 13.9542 & 3.9205 & 0.5921 & -0.0327 \\
\hline 1.4069 & 13.3516 & -5.2691 & 0.2897 & 0.5165 & 2.9569 & 14.1544 & 4.0840 & 0.5902 & -0.0441 \\
\hline 1.4569 & 13.1045 & -4.6239 & 0.3147 & 0.4845 & 2.9677 & 14.1985 & 4.1187 & 0.5897 & -0.0465 \\
\hline 1.5069 & 12.8884 & -4.0320 & 0.3382 & 0.4552 & 2.9785 & 14.2430 & 4.1534 & 0.5891 & -0.0489 \\
\hline 1.5569 & 12.7006 & -3.4867 & 0.3603 & 0.4281 & 2.9892 & 14.2879 & 4.1879 & 0.5886 & -0.0512 \\
\hline 1.6069 & 12.5390 & -2.9824 & 0.3810 & 0.4028 & 3.0000 & 14.3332 & 4.2222 & 0.5880 & -0.0530 \\
\hline 1.6569 & 12.4017 & -2.5140 & 0.4006 & 0.3791 & & & & & \\
\hline
\end{tabular}

\section{Programming}

$u_{1}^{\prime}=u_{2}$

$u_{2}^{\prime}=\frac{1}{8}\left(32+2 t^{3}-u_{1} u_{2}\right)$

$u_{3}^{\prime}=u_{4}$

$u_{3}^{\prime}=-\frac{1}{8}\left(u_{2} u_{3}-u_{1} u_{4}\right)$

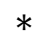

$* *$

$* * *$

$* * * *$

$u_{1}(1)=17 \quad u_{1}(1)=u_{2}(1)=s_{k}$ where $s_{o}=-1.333$ as calculated in step 1

$u_{3}(1)=0 \quad u_{4}(1)=1$ 


\section{Solving using Non - linear shooting method on maple}

We attempt to solve * and $* *$ using the classical $\mathrm{R}-\mathrm{K} 4$ in maple

$>$ Sys $1:=\mathrm{D}(\mathrm{u} 1)(\mathrm{x})-\mathrm{u} 2(\mathrm{x}), \operatorname{Du} 2(\mathrm{x})=\left(32+2^{*} \mathrm{x}^{\wedge} 3-\mathrm{u} 1(\mathrm{x})\right) / 8$

$>\mathrm{a}:=1 ; \mathrm{b}:=3 ; \mathrm{N}:=20 ; \mathrm{h}:=(\mathrm{b}-\mathrm{a}) / \mathrm{N} ;$ alpha: $=17$; beta:=43/3

$>$ init1: = (beta-alpha) / (b-a);

$>\mathrm{g} 1:=$ dsolve $(\{$ sys1,init 1$\}$, numeric, method = classical [rk4], $\{\mathrm{u} 1(\mathrm{x})\}$, stepsize-h);

Now we solve $* * *$ and $* * * *$ using the following command

$>$ sys $2:=\mathrm{D}(\mathrm{u} 3)(\mathrm{x})-\mathrm{u} 4(\mathrm{x}), \mathrm{Du} 4(\mathrm{x}) * \mathrm{u} 3(\mathrm{x}) * \mathrm{u} 4(\mathrm{x})) / 8$

$>\mathrm{a}:=1 ; \mathrm{b}:=3 ; \mathrm{N}: 20 ; \mathrm{h}:=(\mathrm{b}-\mathrm{a}) / \mathrm{N} ;$ alpha: $=43 / 3$

$>$ sk:=(beta-alpha) / (b-a);

$>$ init $1:=\mathrm{u} 1(1)=17, \mathrm{u} 2(1)=\mathrm{sk}, \mathrm{u} 3(1)=0, \mathrm{u} 4(1)=1$;

$>\mathrm{g} 2$ :=dsolve $(\{$ sys2, init 1$\}$, numeric method = classical [rk4], $\{\mathrm{u} 1(\mathrm{x}), \mathrm{u} 2(\mathrm{x}) \mathrm{u} 3(\mathrm{x}), \mathrm{u} 4(\mathrm{x})\}$, stepsize $-\mathrm{h})$;

$>\mathrm{u} 1(\mathrm{x}), \mathrm{u} 2(\mathrm{x}), \mathrm{u} 3(\mathrm{x}), \mathrm{u} 4(\mathrm{x})$

Gives the result in table 1

\section{Solving using Non - Linear finite difference method on maple}

$>$ with(linalg)

$>a:=1 ; b:=3$; alpha: $=17$; beta: $=43 / 3 ; \mathrm{N}:=19 ; \mathrm{h}:=(\mathrm{b}-\mathrm{a}) /(\mathrm{N}+1)$;

$>$ w:=vector $(19,0)$;

$>$ for 1 from 1 to $\mathrm{N}$ do

$>\mathrm{t}[\mathrm{i}]: \mathrm{a}+\mathrm{i}^{*} \mathrm{~h}$

$>\mathrm{w}[\mathrm{i}]:=$ alpha $+\mathrm{i} *($ beta - alpha $) /(\mathrm{b}-\mathrm{a}) * \mathrm{~h}$;

$>$ od;

$>\mathrm{A}:=$ matrix $(19,19,0)$

$>\mathrm{u}:=\operatorname{vector}(19,0)$

$>\mathrm{f}:=(\mathrm{t}, \mathrm{x}, \mathrm{xp})->-\mathrm{xp} / 8$;

$>\mathrm{fxp}:=(\mathrm{t}, \mathrm{x}, \mathrm{xp})->-\mathrm{x} / 8$

The nonzero entries of $\mathrm{H}$ and the right hand side, $\mathrm{u}$ of the linear system are generated as follows:

$>\mathrm{H}[1,1]:=2+\mathrm{h} * \mathrm{~h} * \operatorname{evalf}(\mathrm{fx}(\mathrm{t}[1], \mathrm{w}[1],(\mathrm{w}[2]-$ alpha $) /(2 * \mathrm{~h})))$;

$>\mathrm{H}[1,2]:=-1+\mathrm{h} * \operatorname{evalf}(\mathrm{fxp}(\mathrm{t}[1], \mathrm{w}[1],(\mathrm{w}[2]-$ alpha $) /(2 * \mathrm{~h}))) / 2$;

$>$ for 1 from 2 to $\mathrm{N}-1$ do

$>\mathrm{H}[\mathrm{i}, \mathrm{i}-1]:=-1-\mathrm{h} * \operatorname{evalf}(\mathrm{fxp}(\mathrm{t}[\mathrm{i}], \mathrm{w}[\mathrm{i}],(\mathrm{w}[\mathrm{i}+1]-\mathrm{w}[\mathrm{i}-1]) /(2 * \mathrm{~h}))) / 2$;

$>\mathrm{H}[\mathrm{i}, \mathrm{i}+1]:=-1+\mathrm{h} * \operatorname{evalf}(\mathrm{fxp}(\mathrm{t}[\mathrm{i}], \mathrm{w}[\mathrm{i}],(\mathrm{w}[\mathrm{i}+1]-\mathrm{w}[\mathrm{i}-1]) /(2 * \mathrm{~h}))) / 2$;

$>\mathrm{H}[\mathrm{i}, \mathrm{i}]:=-2+\mathrm{h} * \mathrm{~h} * \operatorname{evalf}(\mathrm{fx}(\mathrm{t}[\mathrm{i}], \mathrm{w}[\mathrm{i}],(\mathrm{w}[\mathrm{i}+1]-\mathrm{w}[\mathrm{i}-1]) /(2 * \mathrm{~h})))$;

$>\mathrm{u}[\mathrm{i}]:=-(-\mathrm{w}[\mathrm{i}-1]+2 * \mathrm{w}[\mathrm{i}]-\mathrm{w}[\mathrm{i}+1]+\mathrm{h} * \mathrm{~h} * \operatorname{evalf}(\mathrm{f}(\mathrm{t}[\mathrm{i}], \mathrm{w}[\mathrm{i}],(\mathrm{w}[\mathrm{i}+1]-\mathrm{w}[\mathrm{i}+1] /(2 * \mathrm{~h}))))$;

$>$ end;

This gives table 2

\section{References}

[1] Williams H Press, Brian P Flannery, Saul A Tuekolsky and Williams T Vetterling. (1986). Numerical Recipes. "THE ART OF SCIENTIFIC COMPUTING" Cambridge University Press, New York.

[2] John C Butcher. (2003). "THE NUMERICAL ANALYSIS OF ORDINARY DIFFERENTIAL EQUATIONS”. John Wiley \& Sons, UK. 
[3] John C Butcher. (1987). "THE NUMERICAL ANALYSIS OF ORDINARY DIFFERENTIAL EQUATIONS". Runge-Kutta and general linear methods. . John Wiley \& Sons, UK.

[4] Mahinder K Jain, satteluri R Iyengar and Rajendra Kumar Jain. (2010). "NUMERICAL METHODS FOR SCIENTIFIC AND ENGINEERING COMPUTATION". 5th edition. New Age International Publishers, Ne Delhi, India.

[5] Arieh Lserles. (1996). "A FIRST COURSE IN THE NUMERICAL ANALYSIS OF DIFFERENTIAL EQUATIONS”, Cambridge University Press.

[6] James Murray Watt. (1976). "MODERN NUMERICAL METHODS FOR ORDINARY DIFFERENTIAL EQUATIONS", clarendon press.

[7] Runge C. (1895). "EXPLICIT 2ND ORDER RUNGE-KUTTA METHOD" Martin Luther University, Halle-Wittenberg, Halle.

[8] Henrici Peter. (1962). "DISCRETE VARIABLE METHODS IN ODES”, New York John Wiley $\&$ Sons.

[9] Leonhard Euler. (1913). "INSTITUTIONAL CALCULI INTEGRALS" primum omnia series prims.

[10] Hubert Harrer, A schuler and E Amelunxen. (1990). "COMPARISON OF DIFFERENT NUMERICAL INTEGRATIONS FOR SIMULATING CELLULOSE NEURAL NETWORKS". In: proceeding of the IEEE International Workshop on cellular neural networks and their Applications, Budapest, p. 151-159.

\footnotetext{
*Corresponding author.

E-mail address: ibrahimismailaomeiza@gmail.com
} 